\title{
Multimorbidity in non-communicable diseases in South African primary healthcare
}

\author{
H Lalkhen, MB ChB, MMed; R Mash, MB ChB, DRCOG, DCH, FRCGP, FCFP, PhD \\ Division of Family Medicine and Primary Care, Faculty of Medicine and Health Sciences, Stellenbosch University, Tygerberg, \\ Cape Town, South Africa
}

Corresponding author: R Mash (rm@sun.ac.za)

\begin{abstract}
Background. Multimorbidity in non-communicable diseases (NCDs) is a complex global healthcare challenge that is becoming increasingly prevalent. In Africa, comorbidity of communicable diseases and NCDs is also increasing.

Objectives. To evaluate the extent of multimorbidity among patients with NCDs in South African (SA) primary healthcare (PHC).

Methods. A dataset obtained from a previous morbidity survey of SA ambulatory PHC was analysed. Data on conditions considered active and ongoing at consultations by PHC providers were obtained.

Results. Altogether 18856 consultations were included in the dataset and generated 31451 reasons for encounter and 24561 diagnoses. Hypertension was the commonest NCD diagnosis encountered (13.1\%), followed by type 2 diabetes (3.9\%), osteoarthritis (2.2\%), asthma (2.0\%), epilepsy (1.9\%) and chronic obstructive pulmonary disease (COPD) (0.6\%). The majority of patients (66.9\%) consulted a nurse and $33.1 \%$ a doctor. Overall $48.4 \%$ of patients had comorbidity and $14.4 \%$ multimorbidity. Multimorbidity (two or more conditions) was present in $36.4 \%$ of patients with COPD, $23.7 \%$ with osteoarthritis, $16.3 \%$ with diabetes, $15.3 \%$ with asthma, $12.0 \%$ with hypertension and $6.7 \%$ with epilepsy. Only $1.1 \%$ also had HIV, $1.0 \%$ TB, $0.4 \%$ depression and $0.04 \%$ anxiety disorders.

Conclusion. About half of the patients with NCDs had comorbidity, and multimorbidity was most common in patients with COPD and osteoarthritis. However, levels of multimorbidity were substantially lower than reported in high-income countries. Future clinical guidelines, training of PHC nurses and involvement of doctors in the continuum of care should address the complexity of patients with NCDs and multimorbidity.

S Afr Med J 2015;105(2):134-138. DOI:10.7196/SAMJ.8696
\end{abstract}

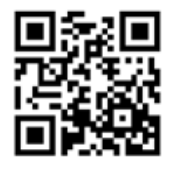

Non-communicable diseases (NCDs) are the leading cause of global mortality, and their prevalence is rising in low- and middle-income countries. ${ }^{[1]}$ This is because of increasing life expectancy, urbanisation and globalisation of the food industry, which drive the four main underlying risk factors of unhealthy diet, physical inactivity, tobacco smoking and harmful use of alcohol. ${ }^{[1]}$

In sub-Saharan Africa, communicable diseases such as HIV, tuberculosis (TB) and malaria have been the leading causes of death in the past. ${ }^{[2]}$ However, because of the prevention and treatment of communicable diseases, particularly HIV, which is now seen as a chronic disease, life expectancy is increasing and enables the emergence of NCDs in middle age. ${ }^{[2]}$ In developing countries, the age of onset of NCDs is younger than in developed countries, and, because of their early age of onset, they lead to more premature deaths. ${ }^{[3]}$ Communicable diseases and NCDs are also interconnected and there is, for example, evidence of a relationship between the treatment of HIV/AIDS and cardiometabolic disorders; between smoking, diabetes and tuberculosis; and between smoking, TB and chronic obstructive pulmonary disease (COPD) ${ }^{[4]}$

The South African (SA) healthcare system faces a quadruple burden of disease, characterised by HIV/AIDS and TB, injury and violence, maternal and child health issues, and NCDs. ${ }^{[2]}$ Currently NCDs are estimated to contribute $28 \%$ to the total burden of disease, and this is predicted to increase substantially over the next few decades if measures are not taken to combat the trend. ${ }^{[2]}$ In the Western Cape, NCDs make up five of the ten leading causes of death: ischaemic heart disease, diabetes, cerebrovascular disease, lung cancer and COPD. ${ }^{[5]}$ The World Health Organization estimates the burden of NCDs to be two to three times higher in SA than in high-income countries. ${ }^{[6]}$ The distribution of NCDs displays socioeconomic disparities, with the heaviest burden among poor communities in urban areas. The rising morbidity and mortality related to NCDs have major implications for the delivery of both acute and chronic healthcare services. ${ }^{[2]}$ In addition, NCDs have economic consequences for individuals, households and society, and are therefore also a developmental challenge. ${ }^{[6]}$

Primary healthcare (PHC) is the foundation of the SA healthcare system and where the majority of patients with NCDs are managed, usually by nurses. ${ }^{[7]}$ The most common NCDs are hypertension, diabetes, asthma, osteoarthritis, COPD and epilepsy. ${ }^{[7]}$ NCDs are generally poorly managed and controlled. ${ }^{[6]}$ Poorly organised healthcare systems, intermittent disruption of drug supplies and lack of capability to manage NCDs on the part of healthcare workers (HCWs) all contribute to patient morbidity and mortality. ${ }^{[8]}$

In high-income countries with elderly populations, the issue of multimorbidity has become a major challenge. In Scotland, for example, over half of the population over the age of 65 years have two or more NCDs. ${ }^{[9]}$ Effective management of multimorbidity and NCDs may require a shift from problem-orientated to goal-orientated care. ${ }^{[10]}$ This means that treatment of patients with multimorbidity should be tailored to each individual patient, that each patient's socioeconomic circumstances and preferences should be taken into account, and that protocols should not be followed blindly. ${ }^{[11]}$ Furthermore, general practitioners believe that using approaches focused on a single disease is not adequate when multiple conditions and their recommended treatments need to be brought together and priorities established, which is a complex task. In people with multimorbidity, some decisions will still be made within a single-disease framework, but decisionmaking will often require balancing competing considerations. ${ }^{[11]}$

At any one moment, there may be a single condition that dominates the clinical picture in a patient with multimorbidity, but over time 
this often changes. Additionally, where a patient has many conditions, single-disease guideline recommendations are sometimes discordant. Different courses of action may be contradictory, for example, prescribing a short course of steroids to a patient with asthma and diabetes, in which situation the multimorbidity creates complexity that requires clinical judgement beyond that offered in guidelines.

In the SA context, there is active debate on how to integrate chronic care for HIV, which is currently organised as a separate vertical service, with TB services and with NCDs. HCWs anticipate a scenario in which large numbers of people have concurrent communicable and non-communicable diseases that need to be treated, and controlled, in an integrated approach. This study, which is based on a national PHC morbidity survey, ${ }^{[7]}$ aimed to measure the current degree of multimorbidity among patients diagnosed with NCDs in SA.

\section{Methods \\ Study design}

The study analysed a dataset obtained from a previous morbidity survey of SA PHC. ${ }^{[7]}$ The design of the study from which the dataset was derived is outlined below.

\section{Setting}

The original study was implemented during 2010 in PHC facilities in the Western Cape, North West, Northern Cape and Limpopo provinces of SA. These provinces were chosen because postgraduate students were available to act as research assistants and because they traversed the country from east to west and north to south. Sequential ambulatory patients who presented to nurses or doctors were included in the study.

\section{Sampling and sample size}

The sample size was based on: $(i)$ the number of HCWs a research assistant could train and support across a number of facilities; and (ii) ensuring that the secondary encounters would occur in large enough numbers. The sample size per province was therefore the product of the number of HCWs that could be handled (60), the number of sampling days for each HCW (5) and the number of patients per day (20), resulting in 6000 encounters per province and 24000 overall.

One district was purposefully selected from each province, based on the location of the research assistants. Out of these districts, four subdistricts were purposively selected, with at least one of the subdistricts an urban area. Urban subdistricts were defined as having a town or metropolitan area and a population of more than 200000 people. In the Western Cape, subdistricts were selected from the metropolitan and West Coast districts to enable a mix of rural and urban populations.

The sample size required from each subdistrict to make up the total of 6000 for the province was stratified according to the population of the subdistrict. The facilities in each subdistrict were then listed and divided into community health centres (CHCs), fixed clinics or mobile clinics. It was assumed that a larger $\mathrm{CHC}$ would have five HCWs participating in the survey, a fixed clinic two and a mobile clinic one. It was also assumed that each $\mathrm{HCW}$ would see at least 20 patients a day and collect data on 5 separate days. The number of HCWs required to deliver the sample size was then determined and distributed between the different types of facilities in proportion to the total number of different facilities in the subdistrict. The required number of $\mathrm{CHCs}$, fixed clinics and mobile clinics were then randomly selected. In the Tygerberg and Klipfontein subdistricts, the City of Cape Town, which runs the clinics, refused permission for the survey and four $\mathrm{CHCs}$ were therefore selected.

\section{Data collection}

At each selected facility, the research assistant explained the project and invited PHC providers (either doctors or nurses) to participate. HCWs were provided with a data collection tool that allowed them to record the age and gender of each patient and up to five reasons for each encounter and five diagnoses for that consultation. No distinction was made between primary and secondary or ongoing diagnoses. HCWs had access to the medical record and recorded all the reasons for the encounter and diagnoses pertinent to the consultation. While chronic conditions that were considered active and ongoing would have been recorded, everything in the past medical history may not have been captured. HCWs were not expected to screen patients for undiagnosed disease. Data were collected on all sequential ambulatory patients seen by the HCW on that day. HCWs were expected to be working in general PHC and not in a specialised vertical programme or emergency department.

\section{Data analysis}

The International Classification of Primary Care, 2nd edition (ICPC-2) ${ }^{[12]}$ was used to code all reasons for encounter and diagnoses. The ICPC-2 was developed by the World Organization of National Colleges, Academies and Academic Associations of General Practitioners/Family Physicians (WONCA) as a classification system uniquely suited to PHC. The system enables classification of the reasons for encounter and diagnoses using a biaxial structure. The first axis codes the body system involved by means of a letter derived from 17 possible chapters. The second axis contains seven components related to different aspects of the consultation. Within each component a menu of standardised rubrics are listed with definitions and inclusion and exclusion criteria. These rubrics provide a two-digit numerical code that is combined with the letter to give the final classification. For example, HIV/AIDS is coded as B90, type 2 diabetes as T90 and tuberculosis as A70.

In this study, the data on NCDs were analysed further with the help of a statistician. Using an Excel spreadsheet, it was possible to analyse the frequency of the following variables for each of the targeted NCDs (hypertension, asthma, COPD, epilepsy and diabetes): age distribution and mean age, gender distribution, other comorbid diseases, and whether the consultation was with a nurse or a doctor. Finally, it was possible to calculate the percentage of patients with different numbers of comorbid conditions for

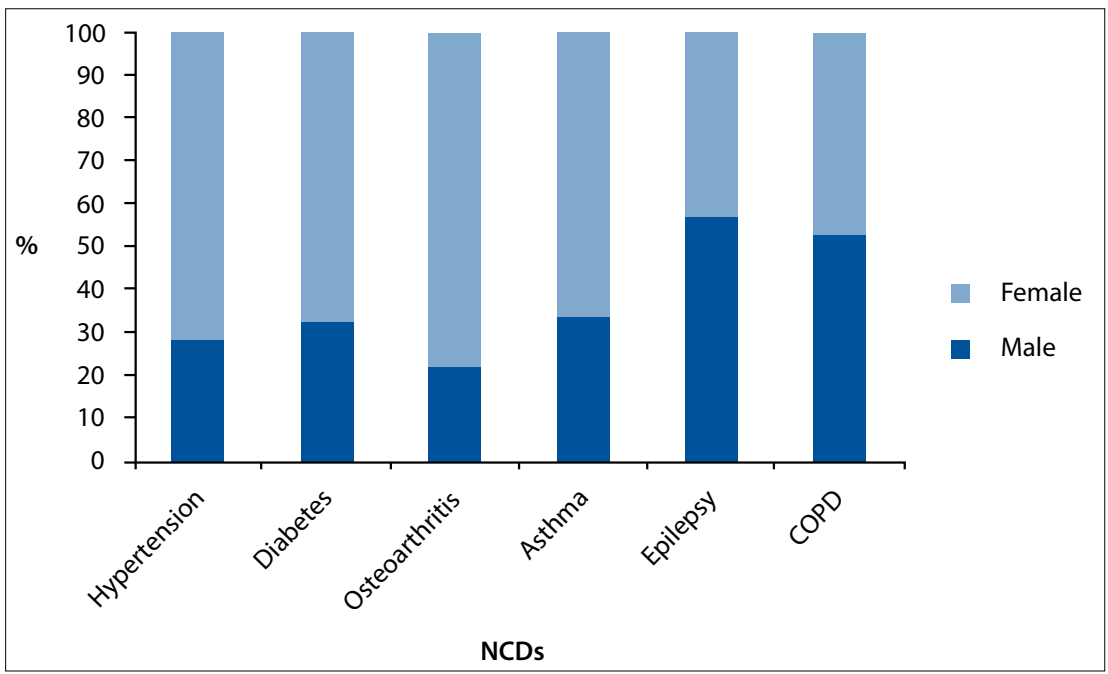

Fig. 1. Gender distribution of patients with selected NCDs. 
each index condition. In this analysis, comorbidity refers to the presence of another disease at the consultation in addition to the selected NCD, while multimorbidity refers to the presence of two or more such diseases.

\section{Results}

Altogether 18856 consultations were included in the survey and generated 31451 reasons for encounter and 24561 diagnoses. Limpopo provided 6678 of the consultations (35.4\%), the Northern Cape 1504 (7.9\%), North West 5082 (26.9\%) and the Western Cape 5592 (29.6\%). Women accounted for $12526(66.6 \%)$ of consultations and men for 6288 (33.4\%).

In the original morbidity study, hypertension was the most common diagnosis encountered in PHC, with 2957 (12\%) having uncomplicated and 262 (1.1\%) complicated hypertension. Another six NCDs were in the top 25 diagnoses seen in PHC: type 2 diabetes 946 (3.9\%), osteoarthritis 530 (2.2\%), asthma $485(2.0 \%)$, epilepsy $375(1.9 \%)$ and COPD 140 (0.6\%). The total number of patients with these NCDs was 5 695, of whom 3811 (66.9\%) were seen by nurses and 1884 (33.1\%) by doctors.

The mean age (standard deviation) of patients with osteoarthritis was 56.9 (13.1) years, while that for COPD was 56.8 (10.1) years, for type 2 diabetes 56.6 (12.9) years, for hypertension 56.4 (13.3) years, for asthma 45.5 (18.1) years and for epilepsy 37.9 (16.4) years. Fig. 1 shows the gender distribution of patients with NCDs. Women were in the majority for hypertension, diabetes and osteoarthritis, whereas there were more men with COPD and epilepsy.

Table 1 illustrates the top ten conditions, in descending order of occurrence, that were comorbid with each of the selected NCDs. Hypertension was the commonest comorbid condition in type 2 diabetes, epilepsy, asthma and COPD. Diabetes and hypertension was the commonest combination.

Table 2 and Fig. 2 show the extent of multimorbidity for each NCD. This table includes patients with type 1 and type 2 diabetes as well as all forms of osteoarthritis. Comorbidity with ICPC codes for other diseases and tumours were included in the calculation, while

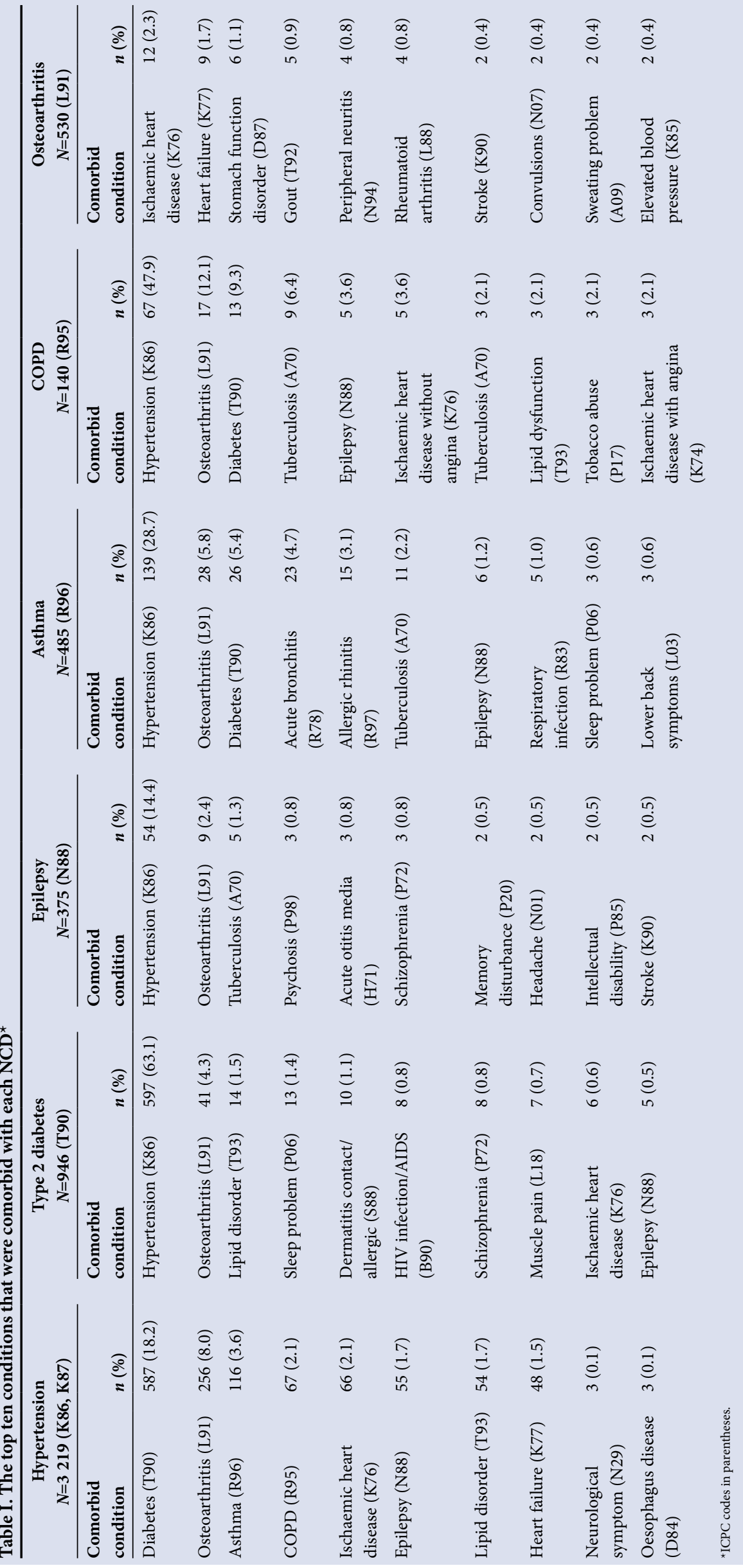


Table 2. Multimorbidity in NCDs

\begin{tabular}{|c|c|c|c|c|c|}
\hline & \multicolumn{5}{|c|}{ Comorbid diseases, $n$} \\
\hline & $\mathbf{0}$ & 1 & 2 & 3 & 4 \\
\hline Hypertension ( $N=3$ 219), $n(\%)$ & $1822(56.6)$ & $1012(31.4)$ & $294(9.1)$ & $89(2.8)$ & $2(0.1)$ \\
\hline Diabetes $(N=999), n(\%)$ & $348(34.8)$ & $488(48.8)$ & $109(10.9)$ & $52(5.2)$ & $2(0.2)$ \\
\hline Asthma $(N=485), n(\%)$ & $265(54.6)$ & $146(30.1)$ & $61(12.6)$ & $13(2.7)$ & $0(0.0)$ \\
\hline Epilepsy $(N=375), n(\%)$ & $283(75.5)$ & $67(17.9)$ & $18(4.8)$ & $7(1.9)$ & $0(0.0)$ \\
\hline COPD $(N=140), n(\%)$ & $51(36.4)$ & $38(27.1)$ & $35(25.0)$ & $15(10.7)$ & $1(0.7)$ \\
\hline Osteoarthritis $(N=575), n(\%)$ & $218(37.9)$ & $221(38.4)$ & $100(17.4)$ & $35(6.1)$ & $1(0.2)$ \\
\hline All $(N=5793), n(\%)$ & $2987(51.6)$ & $1972(34.0)$ & $617(10.7)$ & $211(3.6)$ & $6(0.1)$ \\
\hline
\end{tabular}

infections, injuries and congenital conditions were excluded. Overall, $48.4 \%$ of patients presented with comorbid conditions at the consultation, although the majority (34.0\%) had only one additional condition. Multimorbidity was present overall in $14.4 \%$ of consultations: in $36.4 \%$ of patients with COPD, $23.7 \%$ with osteoarthritis, $16.3 \%$ with diabetes, $15.3 \%$ with asthma, $12.0 \%$ with hypertension and $6.7 \%$ with epilepsy.

Of the patients with NCDs, only $1.1 \%$ were also diagnosed with HIV, $1.0 \%$ with TB, $0.4 \%$ with depression and $0.04 \%$ with anxiety disorders.

\section{Discussion}

Comorbidity was present in almost half of the patients with NCDs and presents a challenge that all HCWs should be aware of. Comorbidity in patients with NCDs ranged from $65.2 \%$ of people with diabetes to $24.5 \%$ of people with epilepsy. Hypertension was the commonest comorbid condition. Multimorbidity was found in $14.4 \%$ of patients, varying from $36.4 \%$ in COPD to $6.7 \%$ in those with epilepsy. Patients of older age had greater levels of comorbidity.

Although multimorbidity is clearly an issue, the extent of the challenge to clinical care is not on the same scale as that in Europe or North America. For example, in Scotland $47 \%$ of patients with diabetes were found to have three or more comorbid NCDs compared with only $5 \%$ of patients in this study, ${ }^{[9]}$ a possible reason being a more elderly Scottish population, with longer life expectancy. Better access to and quality of PHC may also have led to better diagnosis and documentation of comorbid conditions in Scotland. It is likely that many NCDs went undiagnosed in contemporary SA PHC and were therefore not counted, the level of comorbidity reported here almost certainly being an underestimate of the true picture. It is also likely that multimorbidity will increase as SA life expectancy increases.

Mental health disorders such as depression and anxiety were found in $40 \%$ of patients in

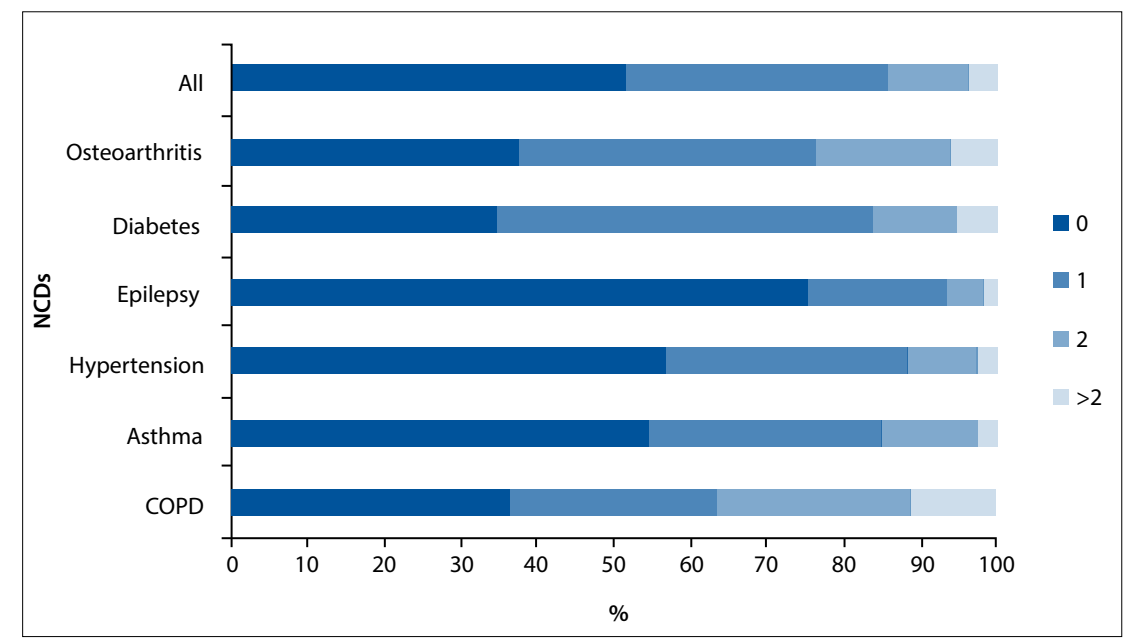

Fig. 2. The extent of multimorbidity in patients with NCDs.

the Scottish study, but were hardly recognised in the SA context. ${ }^{[7]} \mathrm{A}$ recent study of people with hypertension in SA found that $8.1 \%$ had had an anxiety disorder and $4.9 \%$ a depressive disorder during the previous 12 months. ${ }^{[13]}$ Psychological problems also increase in relation to the extent of multimorbidity. ${ }^{[14]}$ The low comorbidity with mental disorders found in this study therefore probably reflects poor ability to recognise these disorders.

Despite concerns in SA about emerging comorbidity between HIV and NCDs, this study suggested that the problem appears very small among patients with NCDs attending ambulatory PHC. In the age group 50 - 60 years, the prevalence of HIV in the population is between $10 \%$ and $15 \%,{ }^{[15]}$ and as the morbidity survey excluded patients with HIV who were being seen in a separate vertical programme, the results cannot shed light on comorbidity among patients with HIV. ${ }^{[7]}$ Notably, there is work that suggests that $30 \%$ of older patients with HIV may have comorbidity with other chronic diseases ${ }^{[16]}$ It is also possible that treatment for NCDs is being given to HIV-positive patients in HIV clinics. Future research is required to investigate these comorbid conditions within the HIV context, especially as HIV-positive patients who were diagnosed in young adulthood mature.

Most patients with $\mathrm{TB}$ are also treated separately and yet would still need to attend ambulatory PHC for treatment of any comorbid NCDs. The current incidence of TB of approximately $1 \%{ }^{[17]}$ would be broadly consistent with the rate found among patients with NCDs in this study.

Multimorbidity increases the complexity of clinical care, as guidelines for different diseases must be integrated and rationalised for management of a specific patient with their own preferences and perspective. Patients should be managed holistically, and all their medical problems attended to by a competent generalist, rather than risk fragmenting care between different services and consultations; patients also deserve their own goals, concerns and preferences to be taken into account. ${ }^{[10]}$ The ability to deliver such care is a challenge, as most are seen by nurses with limited training in the management of NCDs. ${ }^{[18]}$ The PHC system will need to look at guidelines that support a more integrated approach, such as the PC101 PHC guidelines, ${ }^{[19]}$ and the availability of $\mathrm{PHC}$ doctors to assist with more complex patients. ${ }^{[20]}$ 
Improved training, especially when it comes to prescribing, will be necessary as multimorbidity increases. Clinicians often find it difficult to balance the benefits of certain medications with the potential risks of the specific medication or the interaction it may have with existing medications. ${ }^{[21]}$ For example, in patients with hypertension in $\mathrm{PHC}, 73 \%$ of scripts have potential drugdrug interactions, with $7 \%$ of these being potentially severe; in patients with diabetes, $81 \%$ of patients have potential drugdrug interactions, $12 \%$ of which are potentially severe. ${ }^{[21}$ Guidelines take individual conditions into account, but combining recommendations can potentially be harmful in patients with several NCDs. The question whether nurses are adequately trained to manage the potentially complicated patient with numerous NCDs therefore arises. The evidence tends to suggest that nurses/ nurse practitioners do well when using guidelines to manage single diseases, ${ }^{[22]}$ but little evidence exists to suggest the same when it comes to multimorbidity. The role of the family physician may be important in assisting with the clinical care of these patients through consultations, mentoring of nurses/nurse practitioners, and clinical governance activities. ${ }^{[23]}$ Family physicians may offer a more patient-centred and biopsychosocial approach, as a result of their generalist training.

\section{Study limitations}

Not all the provinces in SA were represented in the study, so the findings may not be applicable to the entire population. Districts and subdistricts were not randomly selected, which could influence the results. The sample in the Northern Cape, because of a shortage of staff, was significantly smaller than originally anticipated. The findings are derived from the public sector and are likely to be different in the private sector.

The data represent what has been recognised and documented in PHC and is not necessarily an accurate picture of multimorbidity in the community overall. Some regions in SA are better resourced for the screening and diagnosis of NCDs (e.g. the Western Cape), as a result of which the degree of multimorbidity recognised might be greater than in other areas. The findings represent conditions that had been diagnosed (no screening for undiagnosed conditions was asked for) and were considered to be active and ongoing at that consultation. The results therefore represent the practical comorbidity that needed to be managed, rather than the total comorbidity that might have been present had all conditions been looked for and included.

\section{Implications and recommendations}

- Guidelines for the management of patients with NCDs should take cognisance of the common comorbid conditions found in SA PHC.

- Multimorbidity in patients with NCDs needs to be monitored, as it is likely to increase as life expectancy increases.

- Further research is needed to evaluate the capability of nurses/ nurse practitioners in managing comorbidity and to consider care pathways in PHC that involve doctors appropriately.

- Future research may aim to accurately determine the degree of multimorbidity, when all diseases of interest may be actively looked for under ideal study conditions, as opposed to the pragmatic, reallife conditions employed in this study. This would throw light on the extent to which PHC is currently recognising comorbidity and the quality of care provided.

- Because recognition of mental disorders in patients with NCDs is likely to be low, attention should be given to better recognition, diagnosis and management of such conditions.

\section{Conclusion}

The study set out to determine the prevalence of multimorbidity among patients with common NCDs (diabetes, hypertension, asthma, osteoarthritis, epilepsy, COPD) in SA. Overall $48.4 \%$ of patients consulted had at least one comorbid condition, while $14.4 \%$ had multimorbidity. Findings showed a lower prevalence of multimorbidity relative to that found in high-income countries. Rates of comorbidity ranged from $65.2 \%$ of patients with diabetes to $24.5 \%$ of patients with epilepsy. There was a lower than expected relationship between NCDs and psychiatric conditions. Hypertension was strongly comorbid with diabetes, COPD, asthma and epilepsy. Since patients with NCDs were mostly seen by nurses, future training and guidelines should address the complexity inherent in their consultations. Greater involvement of doctors in managing more complicated patients with multimorbidity should also be considered.

Acknowledgements. RM was the principal investigator in the original primary care morbidity study and made the dataset available. HL analysed the dataset and wrote the initial report. Both authors contributed to and approved the final manuscript. The authors thank Dr Justin Harvey from the Centre for Statistical Consultation at Stellenbosch University for assistance with the analysis.

\section{References}

1. Bradshaw D, Steyn K, Levitt N, Nojilana B. Non-communicable diseases - a race against time. Chronic Disease Initiative for Africa, Department of Medicine, University of Cape Town, 2011. http://www.health. uct.ac.za/usr/health/research/groupings/cdia/downloads/MRC policy brief.pdf (accessed 11 April 2014). 2. Mayosi BM, Flisher AJ, Lalloo UG, Sitas F, Tollman SM, Bradshaw D. The burden of noncommunicable diseases in South Africa. Lancet 2009;374(9693):934-947. [http://dx.doi.org/10.1016/ S0140-6736(09)61087-4]

3. Miszkurka M, Haddad S, Langlois EV, Freeman E, Kouanda E, Zunzunegui M. Heavy burden of noncommunicable diseases at early age and gender disparities in an adult population of Burkina Faso:
constor World health survey. BMC Public Health 2012;12:24. [http://dx.doi.org/10.1186/1471-2458-12-24] World health survey. BMC Public Health 2012;12:24. [http://dx.doi.org/10.1186/1471-2458-12-24]
Van Zyl Smit RN, Pai M, et al. Global lung health: The colliding epidemics of tuberculosis, tobacco 4. Van Zyl Smit RN, Pai M, et al. Global lung health: The colliding epidemics of tuberculosis, tobacco
smoking, HIV and COPD. Eur Respir J2010;35(1):27-33. [http://dx.doi.org/10.1183/09031936.00072909] 5. Naledi T. Progress in NCD programmes in the Western Cape. Presented at the Annual Chronic Disease Initiative for Africa Network Meeting, Cape Town, 11-12 November 2013.

6. Department of Health. South African Declaration on the Prevention and Control of Noncommunicable Diseases. Pretoria: Department of Health, 2011. http://www.doh.gov.za/docs/dcl/2011/ draft_declaration_sa.pdf (accessed 11 April 2014).

Mash B, Fairall L, Adejayan O, et al. A morbidity survey of South African primary care. PLoS One 2012;7(3):1-11. [http://dx.doi.org/10.1371/journal.pone.0032358]

8. Harries A, Jahn A, Zachariah R, Enarson D. Adapting the DOTS Framework for Tuberculosis Control to the management of non-communicable diseases in sub-Saharan Africa. PLoS Med 2008:5(6):859862. [http://dx.doi.org/10.1371/journal.pmed.0050124]

9. Barnett K, Mercer S, Norbury M, Watt G, Wyke S, Guthrie B. Epidemiology of multimorbidity 9. Barnett K, Mercer S, Norbury M, Watt G, Wyke S, Guthrie B. Epidemiology of multimorbidity
and implications for health care, research, and medical education: A cross-sectional study. Lancet and implications for health care, research, and medical education: A cro

10. De Maeseneer J, Boeckxstaens P. James Mackenzie Lecture 2011: Multimorbidity, goal-oriented care, De Maeseneer J, Boeckxstaens P. James Mackenzie Lecture 2011: Multimorbidity, goal-oriented
and equity. Br J Gen Pract 2012;62(600):e522-e524. [http://dx.doi.org/10.3399/bjgp12X652553]

11. Luijks HD, Loeffen MJW, Lagro-Janssen AL, van Weel C, Lucassen PL, Schermer TR. GPs' consideration in multimorbidity management: A qualitative study. Br J Gen Pract 2012;62(600):e503-e510. [http:// dx.doi:10.3399/bjgp12X652373]

12. WONCA International Classification Committee. International Classification of Primary Care (ICPC2). Oxford: Oxford University Press, 1998.

13. Grimsrud A, Stein DJ, Seedat S, Williams D, Myer L. The association between hypertension and depression and anxiety disorders: Results from a nationally-representative sample of South African adults. PLoS One 2009;4(5):e5552. [http://dx.doi.org/10.1371/journal.pone.0005552]

14. Mendenhall E, Richter LM, Stein A, Norris SA. Psychological and physical co-morbidity among urban South African women. PLoS One 2013;8(10):e78803. [http://dx.doi.org/10.1371/journal.pone.0078803]

15. Simbayi LC, Shisana O, Rehle T, et al. South African National HIV Prevalence, Incidence and Simbayi LC, Shisana O, Rehle T, et al. South African National HIV Pron
Behaviour Survey, 2012. Pretoria: Human Sciences Research Council, 2014.

16. Negin J, Martiniuk A, Cumming RG, et al. Prevalence of HIV and chronic comorbidities among older adults. AIDS 2012;26(Suppl 1):S55-S63.
ad

17. Headline TB statistics for South Africa. http://www.tbfacts.org/tb-statistics-south-africa.html (accessed 16 August 2014).

18. Parker W, Steyn NP, Levitt NS, Lombard CJ. They think they know but do they? Misalignment of perceptions of lifestyle modification knowledge among health professionals. Public Health Nutr 2010;14(8):1429-1438. [http://dx.doi.org/10.1017/S1368980009993272]

19. University of Cape Town Lung Institute, Knowledge Translation Unit. Primary Care 101 Integrated Guideline: Symptom-based Approach to the Adult in Primary Care. Cape Town: Department of Health, 2011.

20. Howe AC, Mash RJ, Hugo JFM. Developing generalism in the South African context. S Afr Med J 2013;103(12):899-900. [http://dx.doi.org/10.7196/SAMJ.7509]

21. Kapp PA, Klop AC, Jenkins LS. Drug interactions in primary health care in the George area, South Africa: A cross-sectional study. MMed dissertation. Tygerberg, Cape Town: Stellenbosch University, 2011.

22. Coleman R, Gill G, Wilkinson D. Non-communicable disease management in the resource-poor coleman R, Gill G, Wilkinson D. Non-communicable disease management in the resource-poor
settings: A primary care model from rural South Africa. Bull World Health Organ 1998;76(6):633-640.

23. Mash B. Reflections on the development of family medicine in the Western Cape: A 15-year review. S Afr Fam Pract 2011;53(6):557-562 